\title{
Bonnesen-style symmetric mixed inequalities
}

Pengfu Wang ${ }^{1,2}$, Miao Luo ${ }^{1,3}$ and Jiazu Zhou ${ }^{1 *}$

"Correspondence:

zhoujz@swu.edu.cn

'School of Mathematics and Statistics, Southwest University,

Chongqing, 400715, People's

Republic of China

Full list of author information is

available at the end of the article

\begin{abstract}
In this paper, we investigate the symmetric mixed isoperimetric deficit $\Delta_{2}\left(K_{0}, K_{1}\right)$ of domains $K_{0}$ and $K_{1}$ in the Euclidean plane $\mathbb{R}^{2}$. Via the known kinematic formulae of Poincaré and Blaschke in integral geometry, we obtain some Bonnesen-style symmetric mixed inequalities. These new Bonnesen-style symmetric mixed inequalities are known as Bonnesen-style inequalities if one of the domains is a disc. Some inequalities obtained in this paper strengthen the known Bonnesen-style inequalities.
\end{abstract}

MSC: $52 \mathrm{~A} 10 ; 52 \mathrm{~A} 22$

Keywords: isoperimetric inequality; symmetric mixed isoperimetric deficit; symmetric mixed isoperimetric inequality; Bonnesen-style symmetric mixed inequality

\section{Introductions and preliminaries}

A subset of points $K$ in the Euclidean space $\mathbb{R}^{n}$ is called convex if for all $x, y \in K$, the line segment $\lambda x+(1-\lambda) y(0 \leq \lambda \leq 1)$ joining $x$ and $y$ is contained in $K$. A domain is a set with nonempty interior, and a convex body is a compact convex domain. The Minkowski sum of convex sets $K$ and $L$ is defined by

$$
K+L=\{x+y: x \in K, y \in L\},
$$

and the scalar product of the convex set $K$ with $\lambda \geq 0$ is defined by

$$
\lambda K=\{\lambda x: x \in K, \lambda \geq 0\} .
$$

A homothety of the convex set $K$ is of the form $x+\lambda K\left(x \in \mathbb{R}^{n}, \lambda>0\right)$.

Let $S^{1}$ be the unit circle in $\mathbb{R}^{2}$, and $u \in S^{1}$. The support function $p_{K}(u): S^{1} \rightarrow \mathbb{R}$ of a convex domain $K \subseteq \mathbb{R}^{2}$ is defined by

$$
p_{K}(u)=\max \left\{x \cdot u: x \in K, u \in S^{1}\right\}
$$

and uniquely determines the convex domain $K$. Let $K_{k}(k=0,1)$ be two convex domains of areas $A_{k}$ and perimeters $P_{k}$ in $\mathbb{R}^{2}$. Then

$$
p_{K_{0}}(u) \leq p_{K_{1}}(u) \text { if and only if } K_{0} \subseteq K_{1} .
$$

(c) 2016 Wang et al. This article is distributed under the terms of the Creative Commons Attribution 4.0 International License (http://creativecommons.org/licenses/by/4.0/), which permits unrestricted use, distribution, and reproduction in any medium, provided you give appropriate credit to the original author(s) and the source, provide a link to the Creative Commons license, and indicate if changes were made. 
By the Steiner formula (see [1]) the area of $s K_{0}+t K_{1}$ is

$$
A_{s K_{0}+t K_{1}}=s^{2} A_{0}+2 s t A\left(K_{0}, K_{1}\right)+t^{2} A_{1}
$$

where $A\left(K_{0}, K_{1}\right)$ is called the mixed area of $K_{0}$ and $K_{1}$. The mixed area $A\left(K_{0}, K_{1}\right)$ satisfies (see [1])

$$
A\left(K_{0}, K_{0}\right)=A_{0},
$$

the symmetry

$$
A\left(K_{0}, K_{1}\right)=A\left(K_{1}, K_{0}\right),
$$

the linearity

$$
A\left(K_{0}, s K_{1}+t K_{2}\right)=s A\left(K_{0}, K_{1}\right)+t A\left(K_{0}, K_{2}\right),
$$

and the monotonicity

$$
K_{1} \subseteq K_{2} \quad \Rightarrow \quad A\left(K_{0}, K_{1}\right) \leq A\left(K_{0}, K_{2}\right) .
$$

Let $G_{2}$ be the group of plane rigid motions (see [2-4]), that is, translations and rotations. Let $\theta$ be rotation angle of $K_{1}$ with respect to origin, and $g \in G_{2}$. Then we have (see [4])

$$
\int_{0}^{2 \pi} A\left(K_{0}, g K_{1}\right) d \theta=\frac{1}{2} P_{0} P_{1}
$$

The classical isoperimetric problem says that the disc encloses the maximum area among all plane domains of given perimeter. That is: Let $\Gamma$ be a simple closed curve of perimeter $P$ in the Euclidean plane $\mathbb{R}^{2}$, and $A$ be the area of the domain $K$ enclosed by $\Gamma$; then

$$
P^{2}-4 \pi A \geq 0
$$

where the equality holds if and only if $\Gamma$ is a circle.

The classical isoperimetric problem can root back to Ancient Greece. However, a rigorous mathematical proof of the isoperimetric inequality was obtained during the 19th century (see [2, 5-11]). We can find some simplified and beautiful proofs that lead to generalizations of the discrete case, higher dimensions, the surface of constant curvature, and applications to other branches of mathematics [1, 4, 12-34].

The isoperimetric inequality (1.9) indicates that the quantity

$$
\Delta_{2}(K)=P^{2}-4 \pi A
$$

measures the deficit of domain $K$ and a disc of radius $P / 2 \pi$, and it is called the isoperimetric deficit of $K$. 
During the 1920s, Bonnesen proved a series of inequalities of the form

$$
\Delta_{2}(K)=P^{2}-4 \pi A \geq B_{K},
$$

where $B_{K}$ is a nonnegative invariant of geometric significance and vanishes if and only if $K$ is a disc. An inequality of the form (1.11) is called the Bonnesen-style inequality, and it is stronger than the classical isoperimetric inequality. The following Bonnesen-style inequalities are known.

Proposition 1.1 Let $K$ be a plane domain of area $A$ and bounded by a simple closed curve of perimeter $P$. Denote by $R$ and $r$, respectively, the radius of the minimum circumscribed disc and radius of the maximum inscribed disc of $K$. Then we have

$$
\begin{aligned}
& \pi t^{2}-P t+A \leq 0 ; \quad r \leq t \leq R, \\
& \frac{P-\sqrt{P^{2}-4 \pi A}}{2 \pi} \leq r \leq R \leq \frac{P+\sqrt{P^{2}-4 \pi A}}{2 \pi}, \\
& P^{2}-4 \pi A \geq \pi^{2}(R-r)^{2} .
\end{aligned}
$$

Each equality sign holds when $K$ is a disc.

Many Bonnesen-style inequalities have been found during the past, and mathematicians are still working on unknown Bonnesen-style inequalities of geometric significance. See $[1,3,4,12-16,19-29,32,33,35-46]$ for more references.

Let $K_{k}(k=0,1)$ be two domains of areas $A_{k}$ and bounded by simple closed curves of perimeters $P_{k}$ in $\mathbb{R}^{2}$. Let $d g$ denote the kinematic density of the group $G_{2}$ of plane rigid motions [2-4]. Let $K_{1}$ be convex, and $t K_{1}$ be a dilation of $K_{1}$. Let $n\left\{\partial K_{0} \cap \partial\left(t\left(g K_{1}\right)\right)\right\}$ denote the number of points of intersection $\partial K_{0} \cap \partial\left(t\left(g K_{1}\right)\right)$. Then we have the kinematic formula of Poincaré:

$$
\int_{\left\{g \in G_{2}: \partial K_{0} \cap \partial\left(t\left(g K_{1}\right)\right) \neq \emptyset\right\}} n\left\{\partial K_{0} \cap \partial\left(t\left(g K_{1}\right)\right)\right\} d g=4 t P_{0} P_{1} .
$$

Let $\chi\left(K_{0} \cap t\left(g K_{1}\right)\right)$ be the Euler-Poincaré characteristic of the intersection $K_{0} \cap t\left(g K_{1}\right)$. Then we have the fundamental kinematic formula of Blaschke:

$$
\int_{\left\{g \in G_{2}: K_{0} \cap t\left(g K_{1}\right) \neq \emptyset\right\}} \chi\left(K_{0} \cap t\left(g K_{1}\right)\right) d g=2 \pi\left(t^{2} A_{1}+A_{0}\right)+t P_{0} P_{1} .
$$

If $\mu$ denotes the set of all positions of $K_{1}$ in which either $t\left(g K_{1}\right) \subset K_{0}$ or $t\left(g K_{1}\right) \supset K_{0}$, then the fundamental kinematic formula of Blaschke (1.14) can be rewritten as

$$
\int_{\mu} d g+\int_{\left\{g \in G_{2}: \partial K_{0} \cap \partial\left(t\left(g K_{1}\right)\right) \neq \emptyset\right\}} \chi\left(K_{0} \cap t\left(g K_{1}\right)\right) d g=2 \pi\left(t^{2} A_{1}+A_{0}\right)+t P_{0} P_{1} .
$$

When $\partial K_{0} \cap \partial\left(t\left(g K_{1}\right)\right) \neq \emptyset$, each component of $K_{0} \cap t\left(g K_{1}\right)$ is bounded by at least an arc of $\partial K_{0}$ and an arc of $\partial\left(t\left(g K_{1}\right)\right)$. Therefore, $\chi\left(K_{0} \cap t\left(g K_{1}\right)\right) \leq n\left\{\partial K_{0} \cap \partial\left(t\left(g K_{1}\right)\right)\right\} / 2$. Then by the kinematic formulae of Poincaré (1.13) and Blaschke (1.15) we obtain

$$
\int_{\mu} d g \geq 2 \pi A_{1} t^{2}-P_{0} P_{1} t+2 \pi A_{0} .
$$

Inequality (1.16) immediately gives the following containment theorem [40, 47-49]. 
Containment theorem Let $K_{k}(k=0,1)$ be two domains of areas $A_{k}$ with simple boundaries of perimeters $P_{k}$ in $\mathbb{R}^{2}$. Let $K_{1}$ be convex, and $t K_{1}$ be a dilation of $K_{1}$. A sufficient condition for $t K_{1}$ to contain, or to be contained in $K_{0}$, is

$$
2 \pi A_{1} t^{2}-P_{0} P_{1} t+2 \pi A_{0}>0 .
$$

Moreover, if $t^{2} A_{1} \geq A_{0}$, then $t K_{1}$ contains $K_{0}$.

Let $r_{01}^{g}=\max \left\{t: t\left(g K_{1}\right) \subseteq K_{0} ; g \in G_{2}\right\}$ and $R_{01}^{g}=\min \left\{t: t\left(g K_{1}\right) \supseteq K_{0} ; g \in G_{2}\right\}$ be the inradius of $K_{0}$ with respect to $K_{1}$ and the outradius of $K_{0}$ with respect to $K_{1}$, respectively. It is obvious that $r_{01}^{g} \leq R_{01}^{g}$. Since both $r_{01}^{g}$ and $R_{01}^{g}$ are rigid invariant, we simply call them the relative inradius and the relative outradius and denote them $r_{01}$ and $R_{01}$, respectively. Note that if $K_{1}$ is the unit disc, then the relative inradius $r_{01}$ and the relative outradius $R_{01}$ become the inscribed radius $r$ and the circumscribed radius $R$ of $K_{0}$, respectively.

Note that for $t \in\left[r_{01}, R_{01}\right]$, neither $t K_{1}$ contains $K_{0}$ nor is contained in $K_{0}$. Then by inequality (1.17) we have [40, 47-49]

$$
2 \pi A_{1} t^{2}-P_{0} P_{1} t+2 \pi A_{0} \leq 0, \quad t \in\left[r_{01}, R_{01}\right] .
$$

Inequality (1.18) guarantees that the equation $B_{K_{0}, K_{1}}(t)=2 \pi A_{1} t^{2}-P_{0} P_{1} t+2 \pi A_{0}=0$ has $\operatorname{root}(\mathrm{s})$. Therefore, the determinant of $B_{K_{0}, K_{1}}(t)=0$ is nonnegatitive. Then we have the following symmetric mixed isoperimetric inequality [40, 47-49]:

$$
P_{0}^{2} P_{1}^{2}-16 \pi^{2} A_{0} A_{1} \geq 0
$$

where the equality sign holds if and only if both $K_{0}$ and $K_{1}$ are discs.

When $K_{1}$ is the unit disc, then symmetric mixed isoperimetric inequality (1.19) reduces to the isoperimetric inequality (1.9).

The quantity

$$
\Delta_{2}\left(K_{0}, K_{1}\right)=P_{0}^{2} P_{1}^{2}-16 \pi^{2} A_{0} A_{1}
$$

is called the symmetric mixed isoperimetric deficit of $K_{0}$ and $K_{1}$.

Motivated by the Bonnesen's works in the 1920s, we consider if there is a nonnegative invariant $B_{K_{0}, K_{1}}$ of geometric significance such that

$$
P_{0}^{2} P_{1}^{2}-16 \pi^{2} A_{0} A_{1} \geq B_{K_{0}, K_{1}}
$$

where $B_{K_{0}, K_{1}}$ vanishes if and only if both $K_{0}$ and $K_{1}$ are discs. We call such inequalities Bonnesen-style symmetric mixed inequalities (cf. [40, 47-49]).

The purpose of this paper is to find some new Bonnesen-style symmetric mixed isoperimetric inequalities that strengthen the known Bonnesen-style inequalities.

\section{Bonnesen-style symmetric mixed inequality}

For any two plane domains $K_{k}(k=0,1)$ of areas $A_{k}$ with simple boundaries of perimeters $P_{k}$, the convex hulls $K_{k}^{*}$ of $K_{k}$ increase the areas $A_{k}^{*}$ and decrease the perimeters $P_{k}^{*}$, that is, 
$A_{k}^{*} \geq A_{k}$ and $P_{k}^{*} \leq P_{k}$, so that $P_{0}^{2} P_{1}^{2}-16 \pi^{2} A_{0} A_{1} \geq P_{0}^{* 2} P_{1}^{* 2}-16 \pi^{2} A_{0}^{*} A_{1}^{*}$, that is, $\Delta_{2}\left(K_{0}, K_{1}\right) \geq$ $\Delta_{2}\left(K_{0}^{*}, K_{1}^{*}\right)$. Therefore, the symmetric mixed isoperimetric inequality and Bonnesen-type symmetric mixed inequality are valid for all domains with simple boundaries in $\mathbb{R}^{2}$ if these inequalities are valid for convex domains. Hence, from now on, we only consider convex domains when we estimate the lower bounds of the symmetric mixed isoperimetric deficit.

Lemma 2.1 Let $K_{k}(k=0,1)$ be two convex domains of areas $A_{k}$ and perimeters $P_{k}$ in the Euclidean plane $\mathbb{R}^{2}$. Then

$$
2 \pi A_{1} t^{2}-P_{0} P_{1} t+2 \pi A_{0} \leq 0, \quad t \in\left[r_{01}, R_{01}\right] .
$$

The inequality is strict whenever $r_{01}<t<R_{01}$. When $t=r_{01}$, equality holds if and only if $K_{1}$ is a disc and $K_{0}$ is the Minkowski sum of a disc and a line segment (which may be a point). When $t=R_{01}$, equality holds if and only if $K_{0}$ is a disc and $K_{1}$ is the Minkowski sum of a disc and a line segment (which may be a point).

Proof Let $p_{K_{0}}(u)$ and $p_{\left(g K_{1}\right)}(u)$ are the support functions of convex domains $K_{0}$ and $g K_{1}$, respectively. We can always find $g \in G_{2}$ such that the function $p_{K_{0}}(u)-t p_{\left(g K_{1}\right)}(u)$ about $u$ is nonnegative for $t \in\left[0, r_{01}\right]$. Let $\widetilde{K}_{t}$ be given by

$$
\widetilde{K}_{t}=\left\{x \in \mathbb{R}^{2}: x \cdot u \leq p_{K_{0}}(u)-t p_{\left(g K_{1}\right)}(u) ; u \in S^{1}, g \in G_{2}\right\}, \quad t \in\left[0, r_{01}\right] .
$$

From (2.2) we have that $\widetilde{K}_{0}=K_{0}$ and $\widetilde{K}_{r_{01}}$ is a line segment (which may be a point); see the proof of (6.5.11) in [1]. Therefore,

$$
A\left(\widetilde{K}_{0}\right)=A_{0}, \quad A\left(\widetilde{K}_{r_{01}}\right)=0
$$

From definitions (2.2) and (1.2) we immediately have

$$
\widetilde{K}_{t}+t\left(g K_{1}\right) \subseteq K_{0}
$$

However, relation (2.4), together with the monotonicity (1.7), linearity (1.6), the symmetry of mixed areas (1.5), and (1.4), gives

$$
A\left(K_{0}, g K_{1}\right) \geq A\left(\widetilde{K}_{t}+t\left(g K_{1}\right), g K_{1}\right)=A\left(\widetilde{K}_{t}, g K_{1}\right)+t A\left(g K_{1}\right),
$$

and we have (see the proof of (2.17) in [50]) that

$$
A_{0}-A\left(\widetilde{K}_{t}\right)=2 \int_{0}^{t} A\left(\widetilde{K}_{s}, g K_{1}\right) d s ; \quad t \in\left[0, r_{01}\right]
$$

Now (2.6), (2.5), and (1.8) give

$$
\begin{aligned}
\int_{0}^{2 \pi}\left(A_{0}-A\left(\widetilde{K}_{t}\right)\right) d \theta & =\int_{0}^{2 \pi} 2 \int_{0}^{t} A\left(\widetilde{K}_{s}, g K_{1}\right) d s d \theta \\
& \leq 2 \int_{0}^{2 \pi} \int_{0}^{t}\left(A\left(K_{0}, g K_{1}\right)-s A\left(g K_{1}\right)\right) d s d \theta \\
& =P_{0} P_{1} t-2 \pi A_{1} t^{2}
\end{aligned}
$$


Thus,

$$
2 \pi A_{1} t^{2}-P_{0} P_{1} t+2 \pi A_{0} \leq \int_{0}^{2 \pi} A\left(\widetilde{K}_{t}\right) d \theta .
$$

From (2.5) and (2.7) we see that equality holds in (2.8) if and only if, for all $s \in[0, t]$,

$$
\begin{aligned}
\int_{0}^{2 \pi} A\left(\widetilde{K}_{s}, g K_{1}\right) d \theta & =\int_{0}^{2 \pi}\left(A\left(K_{0}, g K_{1}\right)-s A\left(g K_{1}\right)\right) d \theta \\
& =\frac{1}{2} P_{0} P_{1}-2 \pi s A_{1} .
\end{aligned}
$$

When $t=r_{01}$, by (2.3), (2.8), and (2.9) we have

$$
2 \pi A_{1} r_{01}^{2}-P_{0} P_{1} r_{01}+2 \pi A_{0} \leq 0
$$

where the equality holds if and only if

$$
\int_{0}^{2 \pi} A\left(\widetilde{K}_{r_{01}}, g K_{1}\right) d \theta=\frac{1}{2} P_{0} P_{1}-2 \pi r_{01} A_{1} .
$$

Therefore, we have

$$
2 \pi A_{1} r_{01}^{2}-2\left(\int_{0}^{2 \pi} A\left(\widetilde{K}_{r_{01}}, g K_{1}\right) d \theta+2 \pi r_{01} A_{1}\right) r_{01}+2 \pi A_{0}=0,
$$

that is,

$$
r_{01} \int_{0}^{2 \pi} A\left(\widetilde{K}_{r_{01}}, g K_{1}\right) d \theta=-\pi A_{1} r_{01}^{2}+\pi A_{0}
$$

By (1.3), (2.3), and (2.11) we have

$$
\begin{aligned}
\int_{0}^{2 \pi} A\left(\widetilde{K}_{r_{01}}+r_{01}\left(g K_{1}\right)\right) d \theta & =\int_{0}^{2 \pi}\left(A\left(\widetilde{K}_{r_{01}}\right)+2 r_{01} A\left(\widetilde{K}_{r_{01}}, g K_{1}\right)+A\left(g K_{1}\right) r_{01}^{2}\right) d \theta \\
& =2 \pi A_{0} .
\end{aligned}
$$

Since $\widetilde{K}_{r_{01}}+r_{01}\left(g K_{1}\right) \subseteq K_{0}$, we have $A\left(\widetilde{K}_{r_{01}}+r_{01}\left(g K_{1}\right)\right) \leq A_{0}$. Equality (2.12) forces us to conclude that $A\left(\widetilde{K}_{r_{01}}+r_{01}\left(g K_{1}\right)\right)=A_{0}$ for any $g \in G_{2}$, that is, $\widetilde{K}_{r_{01}}+r_{01}\left(g K_{1}\right)=K_{0}$ for any $g \in G_{2}$. Therefore, $K_{1}$ must be a disc, and $K_{0}$ is the Minkowski sum of a dilation of $K_{1}$ (a disc) and a line segment $\widetilde{K}_{r_{01}}$ (which may be a point).

Let $r_{01}^{\prime}=\max \left\{t: t\left(g K_{0}\right) \subseteq K_{1} ; g \in G_{2}\right\}$ be the inradius of $K_{1}$ with respect to $K_{0}$. Obviously, from the definition of $r_{01}^{\prime}$ and $R_{01}$ it follows that

$$
r_{01}^{\prime}=\frac{1}{R_{01}}
$$

From inequality (2.10) we establish

$$
2 \pi A_{0} r_{01}^{\prime 2}-P_{0} P_{1} r_{01}^{\prime}+2 \pi A_{1} \leq 0
$$


with equality if and only if $K_{0}$ is a disc and $K_{1}$ is the Minkowski sum of a disc and a line segment (which may be a point), that is,

$$
2 \pi A_{1} R_{01}^{2}-P_{0} P_{1} R_{01}+2 \pi A_{0} \leq 0
$$

with equality if and only if $K_{0}$ is a disc and $K_{1}$ is the Minkowski sum of a disc and a line segment (which may be a point).

Finally, inequalities (2.10) and (2.14), together with the well-known properties of quadratic functions, show that

$$
2 \pi A_{1} t^{2}-P_{0} P_{1} t+2 \pi A_{0}<0, \quad r_{01}<t<R_{01} .
$$

Remark 2.1 An analogue of inequality (2.1) can already be found in Bol's work. A complete proof of the analogous inequality (2.1) with equality conditions is given by Böröczky et al. [50] and Luo et al. [51].

When $K_{1}$ is the unit disc, inequality (2.1) reduces to the following known Bonnesen inequality (see $[4,7,20,41])$.

Corollary 2.1 Let $K$ be a convex domain with length $P$ and area $A$ in $\mathbb{R}^{2}$. Denote by $R$ and $r$, respectively, the radius of the minimum circumscribed disc and radius of the maximum inscribed disc of $K$. Then

$$
\pi t^{2}-P t+A \leq 0, \quad t \in[r, R]
$$

The inequality is strict whenever $r<t<R$. When $t=r$, equality holds if and only if $K$ is the Minkowski sum of a disc and a line segment (which may be a point). When $t=R$, equality holds if and only if $K$ is a disc.

Lemma 2.2 Let $K_{k}(k=0,1)$ be two convex domains with areas $A_{k}$ and perimeters $P_{k}$ in $\mathbb{R}^{2}$. Then, for $r_{01} \leq t \leq R_{01}$, we have

$$
P_{0}^{2} P_{1}^{2}-16 \pi^{2} A_{0} A_{1} \geq 4 \pi^{2} A_{1}^{2}\left(R_{01}-t\right)^{2}+\left[2 \pi A_{1}\left(R_{01}+t\right)-P_{0} P_{1}\right]^{2}
$$

The inequality is strict whenever $r_{01}<t<R_{01}$. When $t=r_{01}$, the equality holds if and only if both $K_{0}$ and $K_{1}$ are discs. When $t=R_{01}$, the equality holds if and only if $K_{0}$ is a disc and $K_{1}$ is the Minkowski sum of a disc and a line segment (which may be a point).

Proof By inequality (2.1),

$$
2 \pi A_{1} t^{2}-P_{0} P_{1} t+2 \pi A_{0} \leq 0, \quad t \in\left[r_{01}, R_{01}\right],
$$

so that

$$
2 \pi A_{1} R_{01}^{2}-P_{0} P_{1} R_{01}+2 \pi A_{0} \leq 0
$$


that is,

$$
\begin{aligned}
& -8 \pi^{2} A_{0} A_{1} \geq 8 \pi^{2} A_{1}^{2} t^{2}-4 \pi A_{1} t P_{0} P_{1}, \\
& -8 \pi^{2} A_{0} A_{1} \geq 8 \pi^{2} A_{1}^{2} R_{01}^{2}-4 \pi A_{1} R_{01} P_{0} P_{1} .
\end{aligned}
$$

By adding the last inequalities side by side we have

$$
-16 \pi^{2} A_{0} A_{1} \geq 8 \pi^{2} A_{1}^{2} t^{2}+8 \pi^{2} A_{1}^{2} R_{01}^{2}-4 \pi A_{1} t P_{0} P_{1}-4 \pi A_{1} R_{01} P_{0} P_{1},
$$

that is,

$$
\begin{aligned}
P_{0}^{2} P_{1}^{2}-16 \pi^{2} A_{0} A_{1} \geq & P_{0}^{2} P_{1}^{2}+8 \pi^{2} A_{1}^{2} t^{2}+8 \pi^{2} A_{1}^{2} R_{01}^{2}-4 \pi A_{1} t P_{0} P_{1}-4 \pi A_{1} R_{01} P_{0} P_{1} \\
= & 4 \pi^{2} A_{1}^{2} t^{2}+4 \pi^{2} A_{1}^{2} R_{01}^{2}-8 \pi^{2} A_{1}^{2} t R_{01}+P_{0}^{2} P_{1}^{2}+4 \pi^{2} A_{1}^{2} t^{2} \\
& +4 \pi^{2} A_{1}^{2} R_{01}^{2}+8 \pi^{2} A_{1}^{2} t R_{01}-4 \pi A_{1} t P_{0} P_{1}-4 \pi A_{1} R_{01} P_{0} P_{1} \\
= & 4 \pi^{2} A_{1}^{2}\left(R_{01}-t\right)^{2}+\left[2 \pi A_{1}\left(R_{01}+t\right)-P_{0} P_{1}\right]^{2} .
\end{aligned}
$$

When $t=r_{01}$, the equality holds in (2.16) if and only if the equalities hold in (2.1) when $t=r_{01}$ and $t=R_{01}$, that is, $K_{0}$ and $K_{1}$ are discs. When $t=R_{01}$, the equality holds in (2.16) if and only if the equalities hold in (2.1) when $t=R_{01}$, that is, $K_{0}$ is a disc, and $K_{1}$ is the Minkowski sum of a disc and a line segment (which may be a point). From the equality conditions in (2.1) we know that inequality (2.16) is strict whenever $r_{01}<t<R_{01}$.

Let

$$
f(t)=4 \pi^{2} A_{1}^{2}\left(R_{01}-t\right)^{2}+\left[2 \pi A_{1}\left(R_{01}+t\right)-P_{0} P_{1}\right]^{2}, \quad t \in\left[r_{01}, R_{01}\right]
$$

Then

$$
f^{\prime}(t)=16 \pi^{2} A_{1}^{2}\left(t-\frac{P_{0} P_{1}}{4 \pi A_{1}}\right)
$$

and

$$
f^{\prime \prime}(t)=16 \pi^{2} A_{1}^{2}>0
$$

Therefore, $f(t)$ is concave and reaches the minimum at $t=\frac{P_{0} P_{1}}{4 \pi A_{1}}$ and the maximum at $t=r_{01}$ or $t=R_{01}$. Then we obtain the following Bonnesen-style symmetric mixed inequality.

Corollary 2.2 Let $K_{k}(k=0,1)$ be two convex domains with areas $A_{k}$ and perimeters $P_{k}$ in $\mathbb{R}^{2}$. Then we have

$$
P_{0}^{2} P_{1}^{2}-16 \pi^{2} A_{0} A_{1} \geq 4 \pi^{2} A_{1}^{2}\left(R_{01}-\frac{P_{0} P_{1}}{4 \pi A_{1}}\right)^{2}+\left[2 \pi A_{1}\left(R_{01}+\frac{P_{0} P_{1}}{4 \pi A_{1}}\right)-P_{0} P_{1}\right]^{2}
$$

where the equality holds if and only if both $K_{0}$ and $K_{1}$ are discs. 
Proof When $t=\frac{P_{0} P_{1}}{4 \pi A_{1}}$ in (2.16), we immediately obtain (2.17). From the proof of Lemma 2.2 we see that the equality holds in (2.17) if and only if the equalities hold in (2.1) when $t=R_{01}$ and $t=\frac{P_{0} P_{1}}{4 \pi A_{1}}$, that is, $R_{01}$ and $\frac{P_{0} P_{1}}{4 \pi A_{1}}$ are roots of the equation $B_{K_{0}, K_{1}}(t)=2 \pi A_{1} t^{2}-$ $P_{0} P_{1} t+2 \pi A_{0}=0$. It is obvious that $B_{K_{0}, K_{1}}(t)$ reaches the minimum at $t=\frac{P_{0} P_{1}}{4 \pi A_{1}}$, therefore, there is only one root $R_{01}=\frac{P_{0} P_{1}}{4 \pi A_{1}}$ for the equation $B_{K_{0}, K_{1}}(t)=0$, that is, the determinant $P_{0}^{2} P_{1}^{2}-16 \pi^{2} A_{0} A_{1}=0$. By the symmetric mixed isoperimetric inequality (1.19), $K_{0}$ and $K_{1}$ are discs.

Letting $t=r_{01}$ in inequality (2.16), we immediately obtain the following:

Theorem 2.1 Let $K_{k}(k=0,1)$ be two convex domains with areas $A_{k}$ and perimeters $P_{k}$ in $\mathbb{R}^{2}$. Then we have

$$
P_{0}^{2} P_{1}^{2}-16 \pi^{2} A_{0} A_{1} \geq 4 \pi^{2} A_{1}^{2}\left(R_{01}-r_{01}\right)^{2}+\left[2 \pi A_{1}\left(R_{01}+r_{01}\right)-P_{0} P_{1}\right]^{2}
$$

where the equality holds if and only if both $K_{0}$ and $K_{1}$ are discs.

The following Kotlyar inequality (see [17, 40, 47-49]) is an immediate consequence of Theorem 2.1.

Corollary 2.3 Let $K_{k}(k=0,1)$ be two convex domains with areas $A_{k}$ and perimeters $P_{k}$ in $\mathbb{R}^{2}$. Then we have

$$
P_{0}^{2} P_{1}^{2}-16 \pi^{2} A_{0} A_{1} \geq 4 \pi^{2} A_{1}^{2}\left(R_{01}-r_{01}\right)^{2},
$$

where the equality holds if and only if both $K_{0}$ and $K_{1}$ are discs.

When $t=R_{01}$ in inequality (2.16), we immediately have the following:

Theorem 2.2 Let $K_{k}(k=0,1)$ be two convex domains with areas $A_{k}$ and perimeters $P_{k}$ in $\mathbb{R}^{2}$. Then we have

$$
P_{0}^{2} P_{1}^{2}-16 \pi^{2} A_{0} A_{1} \geq 16 \pi^{2} A_{1}^{2}\left(R_{01}-\frac{P_{0} P_{1}}{4 \pi A_{1}}\right)^{2},
$$

where the equality holds if and only if $K_{0}$ is a disc and $K_{1}$ is the Minkowski sum of a disc and a line segment (which may be a point).

We also have the following:

Lemma 2.3 Let $K_{k}(k=0,1)$ be two convex domains with areas $A_{k}$ and perimeters $P_{k}$ in $\mathbb{R}^{2}$. Then we have

$$
P_{0}^{2} P_{1}^{2}-16 \pi^{2} A_{0} A_{1} \geq 4 \pi^{2} A_{1}^{2}\left(t-r_{01}\right)^{2}+\left[2 \pi A_{1}\left(t+r_{01}\right)-P_{0} P_{1}\right]^{2}, \quad t \in\left[r_{01}, R_{01}\right] .
$$

The inequality is strict whenever $r_{01}<t<R_{01}$. When $t=r_{01}$, the equality holds if and only if $K_{1}$ is a disc and $K_{0}$ is the Minkowski sum of a disc and a line segment (which may be a point). When $t=R_{01}$, the equality holds if and only if both $K_{0}$ and $K_{1}$ are discs. 
Proof By inequality (2.1),

$$
2 \pi A_{1} t^{2}-P_{0} P_{1} t+2 \pi A_{0} \leq 0, \quad t \in\left[r_{01}, R_{01}\right],
$$

and thus

$$
2 \pi A_{1} r_{01}^{2}-P_{0} P_{1} r_{01}+2 \pi A_{0} \leq 0,
$$

so that

$$
\begin{aligned}
& -8 \pi^{2} A_{0} A_{1} \geq 8 \pi^{2} A_{1}^{2} t^{2}-4 \pi A_{1} t P_{0} P_{1}, \\
& -8 \pi^{2} A_{0} A_{1} \geq 8 \pi^{2} A_{1}^{2} r_{01}^{2}-4 \pi A_{1} r_{01} P_{0} P_{1} .
\end{aligned}
$$

By adding the last inequalities side by side we have

$$
-16 \pi^{2} A_{0} A_{1} \geq 8 \pi^{2} A_{1}^{2} t^{2}+8 \pi^{2} A_{1}^{2} r_{01}^{2}-4 \pi A_{1} t P_{0} P_{1}-4 \pi A_{1} r_{01} P_{0} P_{1} .
$$

Then,

$$
P_{0}^{2} P_{1}^{2}-16 \pi^{2} A_{0} A_{1} \geq 4 \pi^{2} A_{1}^{2}\left(t-r_{01}\right)^{2}+\left[2 \pi A_{1}\left(t+r_{01}\right)-P_{0} P_{1}\right]^{2} .
$$

Similarly, following the equality conditions in Lemma 2.2, we have the equality conditions for (2.21).

The function

$$
g(t)=4 \pi^{2} A_{1}^{2}\left(t-r_{01}\right)^{2}+\left[2 \pi A_{1}\left(t+r_{01}\right)-P_{0} P_{1}\right]^{2}, \quad t \in\left[r_{01}, R_{01}\right],
$$

is concave and reaches the minimum at $t=\frac{P_{0} P_{1}}{4 \pi A_{1}}$. Then we immediately obtain the following Bonnesen-style symmetric mixed inequality.

Corollary 2.4 Let $K_{k}(k=0,1)$ be two convex domains with areas $A_{k}$ and perimeters $P_{k}$ in $\mathbb{R}^{2}$. Then we have

$$
P_{0}^{2} P_{1}^{2}-16 \pi^{2} A_{0} A_{1} \geq 4 \pi^{2} A_{1}^{2}\left(\frac{P_{0} P_{1}}{4 \pi A_{1}}-r_{01}\right)^{2}+\left[2 \pi A_{1}\left(\frac{P_{0} P_{1}}{4 \pi A_{1}}+r_{01}\right)-P_{0} P_{1}\right]^{2}
$$

where the equality holds if and only if $K_{0}$ and $K_{1}$ are discs.

Letting $t=R_{01}$ in inequality (2.21), we obtain Theorem 2.1.

When $t=r_{01}$ in inequality (2.21), we have the following Bonnesen-style symmetric mixed inequality.

Theorem 2.3 Let $K_{k}(k=0,1)$ be two convex domains with areas $A_{k}$ and perimeters $P_{k}$ in $\mathbb{R}^{2}$. Then we have

$$
P_{0}^{2} P_{1}^{2}-16 \pi^{2} A_{0} A_{1} \geq 16 \pi^{2} A_{1}^{2}\left(\frac{P_{0} P_{1}}{4 \pi A_{1}}-r_{01}\right)^{2}
$$


where the equality holds if and only if $K_{1}$ is a disc and $K_{0}$ is the Minkowski sum of a disc and a line segment (which may be a point).

The lower bound of symmetric mixed isoperimetric deficit in inequality (2.18) or (2.20) is the maximum of the function $f(t)$. The lower bound of symmetric mixed isoperimetric deficit in inequality (2.18) or (2.23) is the maximum of the function $g(t)$. Which one is the best lower bound of symmetric mixed isoperimetric deficit in inequalities (2.18), (2.20), and (2.23)?

Since

$$
\begin{aligned}
& 16 \pi^{2} A_{1}^{2}\left(R_{01}-\frac{P_{0} P_{1}}{4 \pi A_{1}}\right)^{2} \\
&-\left\{4 \pi^{2} A_{1}^{2}\left(R_{01}-r_{01}\right)^{2}+\left[2 \pi A_{1}\left(R_{01}+r_{01}\right)-P_{0} P_{1}\right]^{2}\right\} \\
&= 8 \pi^{2} A_{1}^{2}\left(R_{01}-r_{01}\right)\left(R_{01}+r_{01}-\frac{P_{0} P_{1}}{2 \pi A_{1}}\right), \\
& 16 \pi^{2} A_{1}^{2}\left(\frac{P_{0} P_{1}}{4 \pi A_{1}}-r_{01}\right)^{2} \\
& \quad-\left\{4 \pi^{2} A_{1}^{2}\left(R_{01}-r_{01}\right)^{2}+\left[2 \pi A_{1}\left(R_{01}+r_{01}\right)-P_{0} P_{1}\right]^{2}\right\} \\
&=-8 \pi^{2} A_{1}^{2}\left(R_{01}-r_{01}\right)\left(R_{01}+r_{01}-\frac{P_{0} P_{1}}{2 \pi A_{1}}\right),
\end{aligned}
$$

and

$$
\begin{gathered}
16 \pi^{2} A_{1}^{2}\left(R_{01}-\frac{P_{0} P_{1}}{4 \pi A_{1}}\right)^{2}-16 \pi^{2} A_{1}^{2}\left(\frac{P_{0} P_{1}}{4 \pi A_{1}}-r_{01}\right)^{2} \\
=16 \pi^{2} A_{1}^{2}\left(R_{01}-r_{01}\right)\left(R_{01}+r_{01}-\frac{P_{0} P_{1}}{2 \pi A_{1}}\right),
\end{gathered}
$$

when $R_{01}+r_{01}-\frac{P_{0} P_{1}}{2 \pi A_{1}} \geq 0$, these lower bounds in inequalities (2.18), (2.20), and (2.23) satisfy

$$
\begin{aligned}
16 \pi^{2} A_{1}^{2}\left(R_{01}-\frac{P_{0} P_{1}}{4 \pi A_{1}}\right)^{2} & \geq 4 \pi^{2} A_{1}^{2}\left(R_{01}-r_{01}\right)^{2}+\left[2 \pi A_{1}\left(R_{01}+r_{01}\right)-P_{0} P_{1}\right]^{2} \\
& \geq 16 \pi^{2} A_{1}^{2}\left(\frac{P_{0} P_{1}}{4 \pi A_{1}}-r_{01}\right)^{2}
\end{aligned}
$$

Therefore, the lower bound $16 \pi^{2} A_{1}^{2}\left(R_{01}-\frac{P_{0} P_{1}}{4 \pi A_{1}}\right)^{2}$ in inequality (2.20) is the best among (2.18), (2.20), and (2.23), that is, inequality (2.20) is the strongest Bonnesen-style symmetric mixed inequality among inequalities (2.18), (2.20), and (2.23).

When $R_{01}+r_{01}-\frac{P_{0} P_{1}}{2 \pi A_{1}} \leq 0$, the lower bounds in inequalities (2.18), (2.20), and (2.23) satisfy

$$
\begin{aligned}
16 \pi^{2} A_{1}^{2}\left(\frac{P_{0} P_{1}}{4 \pi A_{1}}-r_{01}\right)^{2} & \geq 4 \pi^{2} A_{1}^{2}\left(R_{01}-r_{01}\right)^{2}+\left[2 \pi A_{1}\left(R_{01}+r_{01}\right)-P_{0} P_{1}\right]^{2} \\
& \geq 16 \pi^{2} A_{1}^{2}\left(R_{01}-\frac{P_{0} P_{1}}{4 \pi A_{1}}\right)^{2}
\end{aligned}
$$


The Bonnesen-style symmetric mixed inequality (2.23), that is,

$$
P_{0}^{2} P_{1}^{2}-16 \pi^{2} A_{0} A_{1} \geq 16 \pi^{2} A_{1}^{2}\left(\frac{P_{0} P_{1}}{4 \pi A_{1}}-r_{01}\right)^{2}
$$

is the best one among inequalities (2.18), (2.20), and (2.23).

When $K_{1}$ is the unit disc, these Bonnesen-style symmetric mixed inequalities immediately lead to the following known Bonnesen-style inequalities of Burago, Grinberg, Hsiung, Hadwiger, Osserman, Zhou, and Ren (see [9, 12, 16, 20, 21, 41, 44]).

Corollary 2.5 Let $K$ be a convex domain with area $A$ and perimeter $P$ in $\mathbb{R}^{2}$. Denote by $R$ and $r$, respectively, the radius of the minimum circumscribed disc and radius of the maximum inscribed disc of $K$. Then

$$
\begin{aligned}
& P^{2}-4 \pi A \geq(P-2 \pi r)^{2}, \\
& P^{2}-4 \pi A \geq(2 \pi R-P)^{2}, \\
& P^{2}-4 \pi A \geq \pi^{2}(R-r)^{2}+[\pi(R+r)-P]^{2}, \\
& P^{2}-4 \pi A \geq \pi^{2}\left(R-\frac{P}{2 \pi}\right)^{2}+\left[\pi\left(R+\frac{P}{2 \pi}\right)-P\right]^{2}, \\
& P^{2}-4 \pi A \geq \pi^{2}\left(\frac{P}{2 \pi}-r\right)^{2}+\left[\pi\left(\frac{P}{2 \pi}+r\right)-P\right]^{2} .
\end{aligned}
$$

The equality in the first inequality holds if and only if $K$ is the Minkowski sum of a disc and a line segment (which may be a point). The equalities of the other inequalities hold if and only if $K$ is a disc.

\section{Competing interests}

The authors declare that they have no competing interests.

Authors' contributions

All authors read and approved the final manuscript.

\section{Author details}

'School of Mathematics and Statistics, Southwest University, Chongqing, 400715, People's Republic of China. ${ }^{2}$ School of Mathematics and Statistics, Chongqing Technology and Business University, Chongqing, 40067, People's Republic of China. ${ }^{3}$ School of Mathematics and Computer Science, Guizhou Normal University, Guiyang, Guizhou 550001, People's Republic of China.

\section{Acknowledgements}

The corresponding author is supported in part by the NSFC (No. 11271302) and the PhD Program of Higher Education Research Fund (No. 2012182110020). The first author and the second author are supported in part by NSFC (No. 11401486).

Received: 3 January 2016 Accepted: 12 August 2016 Published online: 02 September 2016

\section{References}

1. Schneider, R: Convex Bodies: The Brunn-Minkowski Theory. Cambridge University Press, Cambridge (1993)

2. Blaschké, W: Vorlesungen über Intergral Geometrie, 3rd edn. Deutsch. Verlag Wiss., Berlin (1955)

3. Hadwiger, H: Vorlesungen über Inhalt, Oberfläche und Isoperimetrie. Springer, Berlin (1957)

4. Santaló, LA: Integral Geometry and Geometric Probability. Addison-Wesley, Reading (1976)

5. Banchoff, T, Pohl, W: A generalization of the isoperimetric inequality. J. Differ. Geom. 6, 175-213 (1971)

6. Bokowski, J, Heil, E: Integral representation of quermassintegrals and Bonnesen-style inequalities. Arch. Math. 4, 79-89 (1986)

7. Bonnesen, T: Les problèmes des isopérimètres et des isépiphanes. Gauthier-Villars, Paris (1929)

8. Bottema, O: Eine obere Grenze für das isoperimetrische Defizit ebener Kurven. Ned. Akad. Wet. Proc. 66, 442-446 (1933) 
9. Burago, YD, Zalgaller, VA: Geometric Inequalities. Springer, Berlin (1988)

10. Diskant, V: A generalization of Bonnesen's inequalities. Sov. Math. Dokl. 14, 1728-1731 (1973)

11. Enomoto, K: A generalization of the isoperimetric inequality on $S^{2}$ and flat tori in $S^{3}$. Proc. Am. Math. Soc. 120 553-558 (1994)

12. Grinberg, E, Li, S, Zhang, G, Zhou, J: Integral Geometry and Convexity. Proceedings of the International Conference. World Scientific, Wuhan (2006)

13. Gysin, L: The isoperimetric inequality for nonsimple closed curves. Proc. Am. Math. Soc. 118, 197-203 (1993)

14. Hadwiger, H: Die isoperimetrische Ungleichung in Raum. Elem. Math. 3, 25-38 (1948)

15. Howard, R: The sharp Sobolev inequality and the Banchoff-Pohl inequality on surfaces. Proc. Am. Math. Soc. 126, 2779-2787 (1998)

16. Hsiung, CC: Isoperimetric inequalities for two-dimensional Riemannian manifolds with boundary. Ann. Math. 73, 213-220 (1961)

17. Kotlyar, BD: On a geometric inequality. Ukr. Geom. Sb. 30, 49-52 (1987)

18. Ku, H, Ku, M, Zhang, X: Isoperimetric inequalities on surfaces of constant curvature. Can. J. Math. 49, 1162-1187 (1997)

19. Li, M, Zhou, J: An isoperimetric deficit upper bound of the convex domain in a surface of constant curvature. Sci. China Math. 53, 1941-1946 (2010)

20. Osserman, R: The isoperimetric inequality. Bull. Am. Math. Soc. 84, 1182-1238 (1978)

21. Osserman, R: Bonnesen-style isoperimetric inequality. Am. Math. Mon. 86, 1-29 (1979)

22. Pleijel, A: On konvexa kurvor. Nord. Math. Tidskr. 3, 57-64 (1955)

23. Polya, G, Szegö, G: Isoperimetric Inequalities in Mathematical Physics. Ann. Math. Studies. Princeton University Press, Princeton (1951)

24. Stone, A: On the isoperimetric inequality on a minimal surface. Calc. Var. Partial Differ. Equ. 17, 369-391 (2003)

25. Tang, D: Discrete Wirtinger and isoperimetric type inequalities. Bull. Aust. Math. Soc. 43, 467-474 (1991)

26. Teufel, E: A generalization of the isoperimetric inequality in the hyperbolic plane. Arch. Math. 57, 508-513 (1991)

27. Teufel, E: Isoperimetric inequalities for closed curves in spaces of constant curvature. Results Math. 22, $622-630$ (1992)

28. Wei, S, Zhu, M: Sharp isoperimetric inequalities and sphere theorems. Pac. J. Math. 220, 183-195 (2005)

29. Weiner, J: A generalization of the isoperimetric inequality on the 2-sphere. Indiana Univ. Math. J. 24, 243-248 (1974)

30. Weiner, J: Isoperimetric inequalities for immersed closed spherical curves. Proc. Am. Math. Soc. 120, 501-506 (1994)

31. Yau, ST: Isoperimetric constants and the first eigenvalue of a compact manifold. Ann. Sci. Éc. Norm. Supér. 8, 487-507 (1975)

32. Zhang, X-M: Bonnesen-style inequalities and pseudo-perimeters for polygons. J. Geom. 60, $188-201$ (1997)

33. Zhang, X-M: Schur-convex functions and isoperimetric inequalities. Proc. Am. Math. Soc. 126, 461-470 (1998)

34. Zhang, G, Zhou, J: Containment measures in integral geometry. In: Integral Geometry and Convexity, pp. 153-168. World Scientific, Singapore (2006)

35. Gao, X: A note on the reverse isoperimetric inequality. Results Math. 59, 83-90 (2011)

36. Gao, X: A new reverse isoperimetric inequality and its stability. Math. Inequal. Appl. 15, 733-743 (2012)

37. Pan, $S$, Tang, $X$, Wang, $X$ : A refined reverse isoperimetric inequality in the plane. Math. Inequal. Appl. 13, 329-338 (2010)

38. Pan, S, Zhang, H: A reverse isoperimetric inequality for closed strictly convex plane curves. Beitr. Algebra Geom. 48, 303-308 (2007)

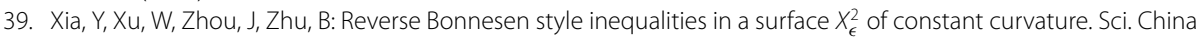
Math. 56, 1145-1154 (2013)

40. Zeng, C, Zhou, J, Yue, S: The symmetric mixed isoperimetric inequality of two planar convex domains. Acta Math. Sin. 55, 355-362 (2012)

41. Zhou, J: Plan Bonnesen-type inequalities. Acta Math. Sin. 50, 1397-1402 (2007)

42. Zhou, J, Du, Y, Cheng, F: Some Bonnesen-style inequalities for higher dimensions. Acta Math. Sin. 28, 2561-2568 (2012)

43. Zhou, J, Ma, L, Xu, W: On the isoperimetric deficit upper limit. Bull. Korean Math. Soc. 50, 175-184 (2013)

44. Zhou, J, Ren, D: Geometric inequalities - from integral geometry point of view. Acta Math. Sci. 30, 1322-1339 (2010)

45. Zhou, J, Xia, Y, Zeng, C: Some new Bonnesen-style inequalities. J. Korean Math. Soc. 48, 421-430 (2011)

46. Zhou, J, Zhou, C, Ma, F: Isoperimetric deficit upper limit of a planar convex set. Rend. Circ. Mat. Palermo (2) Suppl. 81 , 363-367 (2009)

47. Wang, $\mathrm{P}, \mathrm{Xu}, \mathrm{W}, \mathrm{Zhou}, \mathrm{J}, \mathrm{Zhu}, \mathrm{B}$ : On Bonnesen-style symmetric mixed inequality of two planar convex domains. Sci. China Math. 45, 245-254 (2015)

48. $\mathrm{Xu}, \mathrm{W}, \mathrm{Zhou}, \mathrm{J}, \mathrm{Zhu}, \mathrm{B}$ : Bonnesen-style symmetric mixed isoperimetric inequality. In: Real and Complex Submanifolds. Springer Proceedings in Mathematics and Statistics, vol. 106, pp. 97-107 (2014)

49. $\mathrm{Xu}, \mathrm{W}, \mathrm{Zhou}, \mathrm{J}$, Zhu, B: On containment measure and the mixed isoperimetric inequality. J. Inequal. Appl. 2013, 540 (2013)

50. Böröczky, K, Lutwak, E, Yang, D, Zhang, G: The log-Brunn-Minkowski inequality. Adv. Math. 231, 1974-1997 (2012)

51. Luo, M, Xu, W, Zhou, J: Translative containment measure and symmetric mixed isohomothetic inequalities. Sci. China Math. 58, 2593-2610 (2015) 